\title{
Exploring the relationship between governance mechanisms in healthcare and health workforce outcomes: a systematic review
}

\author{
Stephanie E Hastings*, Gail D Armitage, Sara Mallinson, Karen Jackson and Esther Suter
}

\begin{abstract}
Background: The objective of this systematic review of diverse evidence was to examine the relationship between health system governance and workforce outcomes. Particular attention was paid to how governance mechanisms facilitate change in the workforce to ensure the effective use of all health providers.

Methods: In accordance with standard systematic review procedures, the research team independently screened over 4300 abstracts found in database searches, website searches, and bibliographies. Searches were limited to 2001-2012, included only publications from Canada, the United Kingdom, the Netherlands, New Zealand, Australia, and the United States. Peer- reviewed papers and grey literature were considered. Two reviewers independently rated articles on quality and relevance and classified them into themes identified by the team. One hundred and thirteen articles that discussed both workforce and governance were retained and extracted into narrative summary tables for synthesis.
\end{abstract}

Results: Six types of governance mechanisms emerged from our analysis. Shared governance, Magnet accreditation, and professional development initiatives were all associated with improved outcomes for the health workforce (e.g., decreased turnover, increased job satisfaction, increased empowerment, etc.). Implementation of quality-focused initiatives was associated with apprehension among providers, but opportunities for provider training on these initiatives increased quality and improved work attitudes. Research on reorganization of healthcare delivery suggests that changing to team-based care is accompanied by stress and concerns about role clarity, that outcomes vary for providers in private versus public organizations, and that co-operative clinics are beneficial for physicians. Funding schemes required a supplementary search to achieve adequate depth and coverage. Those findings are reported elsewhere.

Conclusions: The results of the review show that while there are governance mechanisms that consider workforce impacts, it is not to the extent one might expect given the importance of the workforce for improving patient outcomes. Furthermore, to successfully implement governance mechanisms in this domain, there are key strategies recommended to support change and achieve desired outcomes. The most important of these are: to build trust by clearly articulating the organization's goal; considering the workforce through planning, implementation, and evaluation phases; and providing strong leadership.

Keywords: Workforce outcomes, Governance, Quality improvement, Work attitudes, Magnet accreditation, Shared governance, Healthcare delivery, Review

\footnotetext{
* Correspondence: stephanie.hastings@albertahealthservices.ca

Alberta Health Services, 10301 Southport Lane SW, Calgary, AB T2W 1S7,
}

Canada

\section{Ciomed Central}

(c) 2014 Hastings et al.; licensee BioMed Central Ltd. This is an Open Access article distributed under the terms of the Creative Commons Attribution License (http://creativecommons.org/licenses/by/2.0), which permits unrestricted use, distribution, and reproduction in any medium, provided the original work is properly credited. 


\section{Background}

Over the last decade, Canadian health systems have undergone numerous changes. The main drivers for change are issues of sustainability [1], perceived health human resource $[\mathrm{HHR}]$ shortages $[2,3]$ and the desire to improve health outcomes $[2,4]$. Significant health system transformation involves changes in structure, processes, culture, and values [5] which better align care with population health needs.

A key element of system transformation is an educated and skilled workforce [1], with appropriate skill mix, that is utilized in an effective and efficient way [6]. Changing the way healthcare providers work together to deliver care may address potential HHR shortages, healthcare provider misdistribution, and help achieve high quality, efficient, and cost-effective care. Furthermore, workforce modification is expected to increase productivity, job satisfaction, recruitment, and retention [7], leading to a sustainable health workforce [8] and more effective and accessible service delivery [9]. However, change impacting the workforce is often limited to the local level if existing organizational governance structures remain intact and reinforce the status quo [10].

Inconsistent definition and operationalization of the concept of governance has been noted [11]. Broadly speaking it encompasses a whole range of structures and processes through which policies (formal and informal) are enacted to achieve goals, including legislation, regulation and oversight, accountability structures, incentives, and policies to set and maintain strategic direction $[12,13]$. In the context of health systems, governance has been characterized as a set of tasks and functions largely established to carry out health ministry goals [14] - essentially driving the direction, type, and accountability of service delivery to improve health system performance [15].

Although there has been increasing interest in governance and health system transformation, as evidenced by a growing literature on the topic, there is still a significant gap in knowledge about how particular 'tools' or mechanisms of governance work, in what context, and how they impact health system actors - particularly the health workforce [11]. Pulling together existing, diverse evidence on governance mechanisms and health workforce outcomes will provide decision makers with a better basis for planning future initiatives [11].

The objective of this systematic review was to increase our understanding of the evidence linking health system governance mechanisms to health workforce outcomes. The research questions guiding the systematic review were:

1. How are workforce outcomes accounted for in governance mechanisms in Canada and internationally?
2. What is the impact of governance mechanisms on health workforce outcomes to support health system change?

3. What elements of governance mechanisms are critical to workforce outcomes?

4. How do health system governance mechanisms facilitate workforce changes and contribute to health system change?

\section{Methods}

Early involvement of an advisory committee composed of decision makers and experts in the fields of healthcare policy, governance, healthcare performance, and health workforce was instrumental to ensure the relevance of the review. They helped shape the research questions, refine the literature search, validate the findings, and identify knowledge dissemination opportunities.

The search strategy was developed in collaboration with a university-affiliated research librarian with extensive knowledge of the healthcare databases. Broad search terms related to governance (e.g., governance, hospital administration, leadership, management, missions, models, health care reform) and workforce (based on a list of clinical occupations) were used to capture as many potentially relevant papers as possible (see Additional file 1 for a sample search strategy; see full report for complete strategy [16]). Eligibility criteria were i) publications discussing health systems in Canada, Sweden, the United Kingdom, the Netherlands, New Zealand, Australia, or the United States, ii) published between 2001 and 2012, iii) English or French language, iv) included health workforce (regulated or unregulated healthcare providers) and included some form of governance. These countries were chosen because each has features similar to the Canadian system, with the exception of the United States. We included American literature to avoid excluding the vast majority of research on governance in healthcare.

The research librarian executed the search strategy. The following databases were searched for peer-reviewed literature: Medline (OVID), Cochrane CENTRAL Register of Controlled Trials (OVID), Health Technology Assessment HTA (OVID), Cochrane Database of Systematic Reviews (OVID), EMBASE (OVID), PsycINFO (OVID), CINAHL (EBSCO), ABI Inform (ProQuest), Business Source Premiere (EBSCO), ERIC (EBSCO). ProQuest Digital Dissertations, Canadian Research Index (ProQuest), Web of Science Conference Citations, Canadian Health Research Collection (Ebrary) were searched for grey literature (i.e., materials found in sources other than traditional peer-reviewed research, such as conference proceedings and government websites). Both manual and Google sitespecific searches of various government and research agency websites were also conducted. 
Abstracts of peer-reviewed and grey literature were screened by four raters for inclusion based on abstract rating criteria (see Additional file 2). These were assigned as follows: Yes* (3 points) to abstracts that definitely informed the research questions and that met the search criteria outlined above, Yes ( 2 points) to abstracts that were likely to inform the research question, Possible (1 point) to abstracts that might possibly inform the research questions, or No (0 points) to abstracts that did not inform the review questions. Inter-rater consistency was established by pre-testing 200 peer-reviewed abstracts and discrepancies were discussed among the four raters until agreement was reached. Following this, we independently screened the entire set of abstracts. Percent agreement among the four raters for the peerreviewed abstracts was $77 \%$ and for grey literature $93 \%$. Raters' scores were summed and full text articles for abstracts scoring at least five points (of a possible 12) were retrieved for further review. Abstracts scoring four points were discussed among the raters to determine inclusion or exclusion. Two researchers reviewed the full text articles for relevancy using the abstract inclusion criteria. If the two raters disagreed on inclusion, articles were discussed among the four abstract raters until agreement was reached.

Articles retained at the screening stage were then categorized using a classification sheet. The classification sheet itemized the country of study, governance mechanism reported, workforce issues discussed and type of research (i.e., empirical, non-empirical), to simplify later extraction.

These articles were read independently by two researchers and rated for quality. The quality-rating criteria for peer-reviewed empirical articles included items pertaining to the methodological soundness of the study (e.g., clear description of the sample, systematic approach to data collection, valid and reliable measures, appropriate analyses and interpretation). Peer-reviewed non-empirical articles and the grey literature were rated on quality of argument, recency, and originality of the ideas discussed. Peer-reviewed empirical and non-empirical quality ratings also included assessments of the risk of bias in each study, based on whether sources of funding were acknowledged and whether at least one author was unaffiliated with the organization under study (one point each). Quality scores were averaged across raters. Empirical articles achieving a minimum average score of 10 (of a possible 17) and non-empirical and grey articles scoring a minimum of 5 (of a possible 10; see Additional file 3 for quality rating sheets) were retained. We considered scores between 15 and 17 points to be high quality, scores between 12.5 and 14.9 points to be medium quality, and articles scoring in the 10-12.4 point range to be a low quality of evidence. In cases where the two researchers' ratings differed by more than three points, a third rater also completed the quality rating and all three scores were averaged. Non-empirical and grey papers scoring 8 points or higher were considered to have high quality evidence, papers scoring 7 to 7.9 were medium quality, and scores between 5 and 6.9 were considered low quality evidence.

Bibliographies of retained articles and systematic reviews were screened and potentially relevant empirical articles were retrieved for screening and quality rating to determine inclusion. Following the abstract and article screening stages, the authors thematically sorted the remaining papers into groups of similar topics for analysis. No articles were excluded at this stage; all articles meeting quality and relevancy criteria were all retained for analysis.

The empirical papers included in the review used diverse research methods. Relevant information was extracted into narrative summary tables created for this review [17]. The tables contained fields for author information, country of interest, level and type of governance, workforce details and outcomes, method, results, and additional information. The narrative summary tables were completed by one researcher and validated by a second researcher. Similarly, narrative summaries of pertinent information from the non-empirical and grey literature were summarized by one researcher and validated by a second researcher (see Additional file 4 for sample nonempirical summaries). Once the extraction templates were fully populated a researcher led the writing of a section of narrative synthesis on an agreed thematic heading and the synthesis was then validated by the team.

\section{Results}

A PRISMA flowchart detailing the numbers of studies at each stage of the literature search is provided in Figure 1.

Although the team used broad search terms to try and identify a wide range of evidence, our requirement that papers should consider governance mechanisms and workforce meant most papers were excluded. In summary, only 113 articles included information on governance mechanisms and workforce and met quality criteria. Forty-nine papers were empirical, 26 were non-empirical, and 38 were grey literature.

\section{Governance mechanisms identified}

Through the review and thematic grouping of papers (as described earlier) six distinct governance mechanisms emerged: shared governance, Magnet accreditation, professional development and education, quality-focused initiatives, reorganization of healthcare delivery, and funding schemes. This article will focus on the first five themes. 


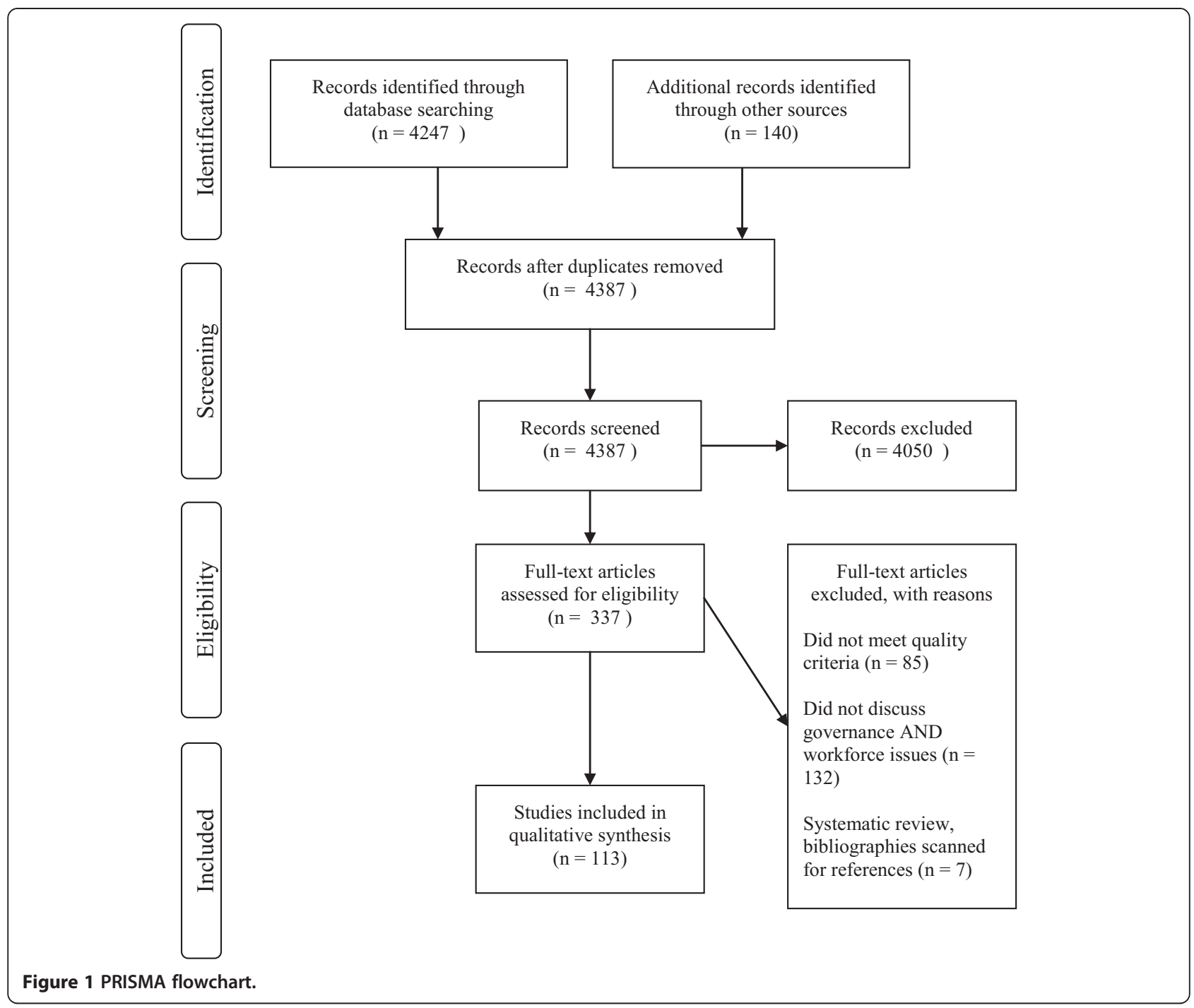

Results for funding schemes will be presented elsewhere due to the large volume of literature on the topic.

\section{Shared governance}

Eight empirical articles measured outcomes of shared governance (see Additional file 5). Three reported shared governance was positively related to empowerment [18-20]. Attree [21] noted that nurses in a facility with few opportunities for professional autonomy (i.e., no shared governance) felt disempowered by their lack of influence over practice. Similar results were found for job satisfaction; Ellenbecker et al. [22] found that shared governance was the only retention strategy employed by a sample of home care agencies that increased satisfaction. Attree [21] found dissatisfaction arising from the lack of a true shared governance structure. Frith and Montgomery [20] found improvements in nurses' relationships with coworkers, physicians, and managers as a result of a shared governance program. Results for retention were less clear; although one study found improvements in retention one year post-implementation [20], another [22] found that shared governance had no effect.

Two empirical studies discussed factors that support the successful implementation of shared governance $[20,23]$. Both noted the importance of support for shared governance from management, along with clear communication between staff and administration. This point was repeated in the non-empirical literature [24-28].

Anderson [24] developed a measure of shared governance that could be used to determine whether shared governance was actually in place. The Index of Professional Nursing Governance [24] was administered in one hospital over time, and results suggested that staff nurses and management were in agreement that the hospital did in fact have a shared governance structure, but that it was not developing at the pace they had anticipated.

Winslow et al. [27] described a five-level clinical laddering program developed by nurses in a shared 
governance program to recognize and reward accomplishments of bedside nurses and motivate them to continue skill development. Although the methodology was not described, they found higher levels of satisfaction among nurses on the advanced levels of the ladder than among nurses on the first two levels. Turnover was substantially lower among nurses on higher levels (less than $1 \%$ for levels 3 to 5 vs. $19 \%$ at levels 1 and 2). Turnover was also reported to be lower among all nurses involved in the laddering program than among those not in the program. Nurses were satisfied with the laddering process itself and appreciated the opportunity to challenge the level at which they were placed based on their practice. Smith Randolph [29], however, tested whether clinical laddering impacted career satisfaction and desire to stay on the job among several allied health professions and found no effect of laddering on either of these two work attitudes.

The quality of papers in the shared governance literature was mid-grade. One empirical paper scored in the high-quality range, three were of low quality, and four were in the medium range. The non-empirical literature, on the other hand, was primarily low quality: four papers scored in the low range, and just one was medium quality.

\section{Magnet accreditation}

Five empirical articles examined Magnet accreditation (see Additional file 6). Four of the articles compared nursing outcomes in Magnet hospitals to those in facilities without such accreditation. Magnet hospitals have higher percentages of RNs and better Safe Practice survey scores than do non-Magnet hospitals [30]. Although the results were not uniformly supportive of the Magnet model's superiority for improving nurse outcomes, the slight majority suggest that Magnet status has certain advantages. Two [31,32] of the three articles examining job satisfaction found higher levels among nurses in Magnet hospitals than among nurses in non-Magnet hospitals. The exception was Hess et al. [33], who found similar satisfaction ratings across Magnet and nonMagnet hospitals. In interviews with senior executives in a hospital applying for Magnet accreditation, Balogh and Cook [34] found evidence of improved staff morale and internal networks. These authors did note, however, that because the interviews were conducted during the accreditation application process, there may be some bias in the results.

Only one article from the grey literature discussed Magnet accreditation. The New Zealand Ministry of Health [35] described an initiative to develop Magnet characteristics in the country's hospitals in order to reduce staff turnover and burnout and improve recruitment, nurse job satisfaction, and nurse injury rates.
Information about the success of the initiative was not available.

The empirical papers that discussed Magnet accreditation were of mixed quality. One paper scored in the high range, two were medium, and two were low quality. The grey article included in this section was in the low range for quality.

\section{Professional development and education}

Seven empirical articles discussed professional development and education programs (see Additional file 7). In general, the training programs resulted in positive outcomes, except in a study by Smith Randolph [29] which found no effect of continuing education on career satisfaction or desire to stay on the job. MacDonald et al. [36] studied a training course designed to enhance collaborative practice. Most learners felt the course increased their confidence in collaborative practice, helped them apply new skills and knowledge in the workplace, and improved collaborative practice. However, there was no change in team members' attitudes toward teamwork. Garrard et al. [37], while studying a nationwide Hepatitis $\mathrm{C}$ training program, found increases in knowledge and confidence about screening, diagnosis, treatment, and patient follow-up. These authors also surveyed participants at one, three, and six months post-training and found that all 28 sites reported at least one major change after one month (e.g., increased communication and collaboration with mental health staff) and that by six months, more than half the sites reported continued improvements in treatment protocols. George et al. [38] examined a training program intended to improve shared leadership in nurses. Positive results were found in pre- and post-program self- and peer-assessments of leadership behaviours in a sample of nurses across five hospitals. Interviews in the months following the training revealed that nurses felt more capable of meeting patient needs and promoting faster recovery, as well as an increased sense of personal growth. They also saw themselves as resources for other staff after the training and noted that better coworker relationships had developed as a result of workflow changes after the training.

The effectiveness of continuing education mandates was the subject of two empirical studies. Results were somewhat conflicting; Prater and Neatherlin [39] surveyed nurses with mandatory continuing education requirements and found they attributed a significant portion of their improvements in various skills to participation in mandatory training. They also had a generally positive view of mandatory continuing education. Smith [40], on the other hand, compared nurses with and without continuing education mandates and found very few meaningful differences in self-rated ability, growth in professional abilities, or hours spent in relevant continuing education courses. Nurses with and 
without continuing education mandates in this study also made very similar attributions about the sources of their professional growth.

McCabe and Garavan [41] examined the effects of organizational support for staff training and found that nurses' commitment and motivation were improved by administrative support of training. The non-empirical and grey literature reinforced this point. Narayanasamy and Narayanasamy [42] discussed staff development programs in the UK's National Health Service (NHS), noting that the organization needs to be supportive, fair, and transparent about staff development and that development plans should be based on accurate appraisals of employee needs. They also argued that development policies should be harmonized with NHS strategies for staff development in order to create a truly supportive organizational culture.

Only one empirical paper discussing professional development scored in the medium range; the remainder were in the low range of acceptability for inclusion in this review. All the non-empirical and grey literature papers were in the low range.

\section{Quality-focused initiatives}

Fifteen empirical articles on quality-focused initiatives (see Additional file 8), which include clinical governance, evidence-based practice, or quality improvement initiatives, were included. Many of these examined providers' attitudes toward the initiatives. In general, providers were supportive of quality initiatives [43-48], although there was often some apprehension about its implementation $[43,45,49]$. Two articles found that quality initiatives changed workloads; one noted a decrease [48] whereas the other noted a significant increase [47]. Only one study [50] found a large percentage of providers namely dentists - with negative attitudes about a quality initiative. Dentists in the UK felt they were lacking guidance, that costs and time demands were too high, and that the costs of the initiative would encourage dentists to leave the NHS to practice privately. Interestingly, however, only $30 \%$ of the dentists surveyed agreed that care quality would not be improved by the program.

Five of the included studies examined the effects of training on attitudes towards or understanding of quality initiatives. Four of these found that training increased acceptance and understanding of quality initiatives [44,51-53]. Sweeney and Ellis [44] also found enhanced leadership skills and better team relationships after training. Levin et al. [51] and Wallen et al. [52] report that training programs increased providers' use of evidence-based practice. However, it must also be noted that these training programs were associated with increased workload [44,45], stress [44], and time pressure [44].
Factors found to facilitate implementation of quality improvement programs are the availability of credible evidence [49,54], the ease of use of the new practice [54], and, most commonly, leadership support $[48,49,52,55]$.

Facilitators of quality initiatives were also found in the non-empirical and grey literature. Leadership and organizational support were noted as being critical to successful introduction and ongoing use [56-62]. Other methods of engaging staff in quality improvement initiatives were to give them some ownership in the program and hold them accountable for its success $[56,57,61,63]$, ensure that adequate information and resources are provided $[57,59,64]$, and, as also suggested by the empirical results, provide staff with adequate training [64-66]. Offering incentives to providers to meet quality standards was also suggested as a means to change provider behaviour $[64,65]$. The importance of including a performance review process in any quality improvement initiative was emphasized by several authors $[63,64,66,67]$.

Engaging physicians in quality improvement initiatives can be difficult $[60,67]$. Some aspects of medical culture are not conducive to quality improvement, such as physicians' traditional separation from other care providers [67], and physicians may fear a loss of autonomy, power, and status if they are pushed into interdisciplinary care teams as part of quality improvement projects [67]. These authors also argue that physicians are reluctant to follow clinical guidelines as this might inhibit clinical freedom and devalue clinical judgment. Shortt et al. [65] noted that while Canadian physicians are distrustful of healthcare reform attempts by the government, they generally support quality improvement. The challenge, then, is to reconcile these two viewpoints. Reinertsen et al. [60] created a framework to engage physicians in quality projects: hospitals must link their quality agendas to the physicians' own quality agenda, recognizing that both parties do want the best quality of care. Physicians must be held responsible for quality, and thus specific roles must be played by physicians and they should be involved from the beginning in planning and implementation. These authors also recommended that hospitals should "standardize what is standardizable, no more" (p. 20), and not create complex care protocols with multiple branches for every possible aspect of care. This was echoed by Mohide and Coker [56], who suggested that evidence-based changes to nursing practice need to be readily understandable, practical, and easy to apply.

Common to the non-empirical literature is a sense that quality-focused initiatives have an impact on providers' feelings of empowerment to make decisions $[58,59,68,69]$. Quality improvement is also touted as a means to increase retention [68] and providers' potential for growth [58] by 
matching staff's knowledge and capabilities with their work, thus encouraging better engagement of clinicians and more effective ways of working [68].

Quality of evidence in the empirical papers focusing on care quality initiatives was somewhat low. Eight of the papers were in the low range, four were medium, and three were of high quality. All but two of the nonempirical and grey papers were considered to be low quality, with the others reaching only medium quality.

\section{Reorganization of healthcare delivery}

Ten empirical articles examined various aspects of the structure of healthcare delivery (see Additional file 9). Three of these studies discussed the change from care delivered by individual providers to team-based care delivery, and the results suggest that providers have difficulties adjusting to the change. Belling et al. [70] found that professionals involved in interdisciplinary mental health teams in the UK experienced anxiety about role changes and role overlap resulting from a collaborative care model. Lavoie-Tremblay et al. [71] examined interdisciplinary teams in two psychiatric hospitals in Quebec and found that, although providers believed the interdisciplinary teamwork was rewarding and allowed them more flexibility in their practice, there was also an increase in psychological distress associated with the move to team-based care. Expected improvements in outcomes such as social support from superiors, use of evidence, balance between effort expended and rewards received, and workload did not appear, although providers in one of the hospitals did note an improvement in social support from colleagues. Sicotte et al. [72] examined the factors that contribute to intensity of interdisciplinary collaboration in Quebec's Community Health Care Centres and found that almost none of the structural or managerial characteristics of the program had any effect. Instead, the most important determinants of collaboration were intragroup process variables such as conflict, belief in benefits of collaboration, and social integration within groups.

O'Dowd et al. [73] examined physicians' work attitudes as a result of a move to co-operative services to cover work outside of normal hours. Most physicians reported improvements to their quality of life, stress levels, and ability to cope with the demands of work. They were also quite satisfied with the other co-op staff, the shift allocation method, independence, and their own confidence for out-of-hours work. However, half of physicians felt overburdened by co-op responsibilities. Almost twothirds of respondents would prefer a physician-health board partnership be responsible for organization of care, compared to $23 \%$ who would prefer the general practitioner take primary responsibility.
Silvestro and Silvestro [74] examined nurse scheduling practices and their effects on nurses. They identified increased staff stress, work-family conflict, low morale, and poor staff-management relations as potential outcomes of poorly designed schedules. They also found that absenteeism, turnover, and difficulties with recruitment could result from improperly designed scheduling processes.

Braithwaite and Westbrook [75] surveyed staff attitudes toward clinical directorates (organizational arrangements through which specific parts of larger hospitals are managed, e.g., medical, surgical, cardiac services) in an Australian hospital. These authors found large variation and uncertainty in staff attitudes, and concluded that staff were unsure about governance in the hospital and were not clear about the purpose, contribution, or effects of clinical directorates.

The remainder of the articles examined differences across organization types. Donoghue and Castle [76] and Castle and Engberg [77] measured the effects of nursing home features on nurse retention and found opposing results. Donoghue and Castle found that for-profit status lowered turnover for Licensed Practical Nurses (LPNs) but not Registered Nurses (RNs) or Nursing Aides (NAs), while membership in a nursing home chain was associated with higher turnover for RNs and LPNs but not for NAs. However, Castle and Engberg found no relation between chain membership and turnover in any group of nurses, but not-for-profit status was associated with lower staff turnover for all nurse types. Castle and Engberg also examined the effect of top management turnover on nursing turnover, and found that NAs and RNs (but not LPNs) were more likely to leave when top management turnover was high.

Aarons et al. [78] examined mental health providers' use of and beliefs in evidence-based practice (EBP) in private versus public agencies. Workers in private agencies were more supportive of EBP than were workers in public agencies, but this did not predict actual use of EBP. Organizational support for EBP, however, did increase its use. This was true across agency types, although private agencies tended to be more supportive of EBP.

The non-empirical and grey literature contained six articles relevant to reorganization of healthcare delivery. Three of these examined physician organizations in the USA. Smith [79] discussed how Accountable Care Organizations impact the quality and type of care physicians provide. Physicians, according to Smith, are often caught in the middle between cutting costs and avoiding liability because the standard of care set by the government does not take into account the realities of cost cutting. Korda and Eldridge [80] discussed the implications of Accountable Care Organizations for nurses, noting that the ability to participate in interprofessional 
care and teamwork will be of crucial success. Howard [81] described a physician organization's difficulties with building a large physician network to sustain a multi-state healthcare system. Difficulties arose immediately, but over time, administration realized that they needed to understand the issues facing physicians (e.g., increased workload needed to maintain results, inflation of practice overhead) and began to place new emphasis on items valued by physicians to build trusting, honest relationships.

Research has shown that incivility and other counterproductive behaviours in the workplace have a significant and negative impact on employees, and so Holloway and Kusy [82] developed a Toxic Organization Change System to reduce and monitor this type of behaviour. The system includes policies, standards, review, and education to address toxicity at the organization, team, and individual levels. Importantly, the authors note that incivility will not stop based on simple education programs or termination of offenders; systematic, multi-level, coordinated strategies are required.

Scott and Lagendyk [83] examined interprofessional relationships in primary care networks in Alberta. They studied five such networks and found that geographical co-location, strong leadership, effective communication strategies, and trust were important to good relationships. These relationships, in turn, were crucial for success in quality improvement initiatives and other practice changes.

Hinings et al. [84] discussed the uncertainty involved in system transformations such as the move to regional health systems in Alberta in 1995. They argued that regionalization had substantial impacts on professional identity for healthcare providers, noting that moves to team-based work and changes to professional boundaries involved changes to what providers did, how they were rewarded, and, consequently, how providers saw themselves.

The quality of evidence on the topic of organization of healthcare delivery was relatively low. The majority of empirical papers scored in the low range, two scored in the medium range, and two were considered high quality. Of the non-empirical and grey literature, two papers were considered medium quality and the remainder was in the low quality range.

\section{Discussion}

The purpose of this systematic review was to examine the evidence relating governance mechanisms in healthcare to health workforce outcomes. We identified six governance mechanisms in the empirical literature: shared governance, Magnet accreditation, professional development initiatives, quality-focused initiatives, reorganization of health services (e.g., moves to team-based care, private vs. public organizations), and funding models. Results on the funding models will be reported elsewhere. Responses to our specific research questions are below.
How are workforce outcomes accounted for in governance mechanisms in Canada and internationally?

Workforce variables were taken into account to varying degrees in emerging governance mechanisms. Table 1 lists the articles reporting relationships between workforce outcomes and governance mechanisms in the empirical literature. A substantial portion of the literature was devoted to examining various work attitudes (e.g., job satisfaction, engagement) in relation to governance mechanisms. Recruitment and retention were also examined, but perhaps to a surprisingly small extent given the workforce shortages some organizations have forecasted for the coming years [7]. Collaborative practice issues were studied primarily in relation to clinical governance, and absenteeism was examined in only a handful of studies. The same was true for role clarity, learning, workload, and skill or staff mix.

These patterns suggest that researchers are missing opportunities to study aspects of health workforce outcomes (e.g., recruitment, collaborative practice) that could be important for the sustainability of healthcare systems and the quality of patient outcomes. The reverse is also true; decisions about new governance mechanisms do not seem to be influenced by research findings as often as they could or should be.

An important finding of our review is that workforce outcomes are often not explicitly considered in governance mechanism planning efforts. Many of the articles we examined were written by academic researchers studying an initiative after its planning phase, rather than by planners intentionally including the impact to the workforce as a factor in the design of governance mechanisms. The majority of initiatives seemed to be ultimately aimed at improving patient outcomes or reducing financial costs (both worthy goals, of course), not explicitly at improving HHR outcomes. Changes for the workforce are implicit in the planning phase (e.g., implementation of a quality initiative will impact how providers work, but the true goal is to improve patient care) but do not seem to be considered in their own right.

What is the impact of governance mechanisms on health workforce outcomes to support health system change? Most governance mechanisms identified during this systematic literature review had at least some of the intended effects on workforce outcomes. Shared governance, Magnet accreditation, and professional development initiatives were most consistently associated with increases to empowerment, confidence, and job satisfaction. Although retention was thought to improve with these initiatives, turnover was not well-studied; shared governance had mixed results and no studies measured the impact of Magnet accreditation or professional development on turnover. However, the significant link 
Table 1 Outcomes examined in empirical articles

\begin{tabular}{|c|c|c|c|c|c|}
\hline Outcome examined & $\begin{array}{c}\text { Shared } \\
\text { governance }\end{array}$ & $\begin{array}{c}\text { Magnet } \\
\text { accreditation }\end{array}$ & $\begin{array}{c}\text { Professional } \\
\text { development }\end{array}$ & $\begin{array}{c}\text { Quality-focused } \\
\text { initiatives }\end{array}$ & $\begin{array}{c}\text { Reorganization of } \\
\text { healthcare delivery }\end{array}$ \\
\hline Absenteeism & & & & Som (2007) [53] & Silvestro (2008) [74] \\
\hline \multirow[t]{4}{*}{$\begin{array}{l}\text { Adoption of } \\
\text { care protocols }\end{array}$} & & $\begin{array}{l}\text { Jayawardhana } \\
\text { (2011) [30] }\end{array}$ & Garrard (2006) [37] & Gerrish (2008) [49] & $\begin{array}{l}\text { Lavoie-Tremblay } \\
\text { (2011) [71] }\end{array}$ \\
\hline & & & & Levin (2011) [51] & \\
\hline & & & & Melnyk (2010) [46] & \\
\hline & & & & Wallen (2010) [52] & \\
\hline \multirow{3}{*}{$\begin{array}{l}\text { Collaborative } \\
\text { practice }\end{array}$} & & Balogh (2006) [34] & Garrard (2006) [37] & Fitzgerald (2003) [54] & Sicotte (2002) [72] \\
\hline & & & George (2002) [38] & Rosengren (2012) [48] & \\
\hline & & & MacDonald (2008) [36] & Som (2007) [53] & \\
\hline \multirow[t]{2}{*}{ Learning } & & & Garrard (2006) [37] & Luxford (2011) [55] & Prater (2001) [39] \\
\hline & & & MacDonald (2008) [36] & Fitzgerald (2003) [54] & Smith (2004) [40] \\
\hline \multirow{4}{*}{$\begin{array}{l}\text { Professional } \\
\text { behaviour }\end{array}$} & Latham (2011) [23] & Balogh (2006) [34] & George (2002) [38] & Freeman (2004) [85] & Aarons (2009) [78] \\
\hline & & & & Paxton (2006) [86] & Prater (2001) [39] \\
\hline & & & & Rondeau (2007) [69] & Smith (2004) [40] \\
\hline & & & & Sheaff (2004) [47] & \\
\hline Recruitment & Latham (2011) [23] & & & Som (2007) [53] & Silvestro (2008) [74] \\
\hline \multirow[t]{4}{*}{ Retention } & $\begin{array}{l}\text { Ellenbecker } \\
\text { (2007) [22] }\end{array}$ & $\begin{array}{l}\text { Brady-Schwartz } \\
\text { (2005) [32] }\end{array}$ & & Levin (2011) [51] & Castle (2006) [77] \\
\hline & Latham (2011) [23] & & & McCormick (2006) [50] & Donoghue (2009) [76] \\
\hline & & & & Som (2007) [53] & Silvestro (2008) [74] \\
\hline & & & & Wallen (2010) [52] & \\
\hline Role clarity & & & MacDonald (2008) [36] & & Belling (2011) [70] \\
\hline \multirow[t]{2}{*}{ Skill/staff mix } & & $\begin{array}{l}\text { Jayawardhana } \\
\text { (2011) [30] }\end{array}$ & & & McCloskey (2005) [87] \\
\hline & & & & & Silvestro (2008) [74] \\
\hline \multirow[t]{13}{*}{ Work attitudes } & Attree (2005) [21] & Balogh (2006) [34] & Garrard (2006) [37] & Dean (2004) [45] & Aarons (2009) [78] \\
\hline & Barden (2011) [18] & $\begin{array}{l}\text { Brady-Schwartz } \\
\text { (2005) [32] }\end{array}$ & George (2002) [38] & Freeman (2004) [85] & Braithwaite (2004) [75] \\
\hline & $\begin{array}{l}\text { Ellenbecker } \\
\text { (2007) [22] }\end{array}$ & Hess (2011) [33] & MacDonald (2008) [36] & Gerrish (2008) [49] & $\begin{array}{l}\text { Lavoie-Tremblay } \\
\text { (2011) [71] }\end{array}$ \\
\hline & Erickson (2003) [19] & Upenieks (2003) [31] & McCabe (2008) [41] & Levin (2011) [51] & O'Dowd (2006) [73] \\
\hline & Frith (2006) [20] & & $\begin{array}{l}\text { Smith Randolph } \\
\text { (2005) [29] }\end{array}$ & Luxford (2011) [55] & Prater (2001) [39] \\
\hline & Kramer (2008) [88] & & & McCormick (2006) [50] & Silvestro (2008) [74] \\
\hline & Latham (2011) [23] & & & Melnyk (2010) [46] & \\
\hline & $\begin{array}{l}\text { Smith Randolph } \\
\text { (2005) [29] }\end{array}$ & & & Murray (2004) [43] & \\
\hline & & & & Rondeau (2007) [69] & \\
\hline & & & & Rosengren (2012) [48] & \\
\hline & & & & Sheaff (2004) [47] & \\
\hline & & & & Sweeney (2003) [44] & \\
\hline & & & & Wallen (2010) [52] & \\
\hline Workload & & $\begin{array}{l}\text { Jayawardhana } \\
\text { (2011) [30] }\end{array}$ & & Rosengren (2012) [48] & McCloskey (2005) [87] \\
\hline
\end{tabular}


between job satisfaction and turnover in BradySchwartz's [32] study of Magnet accreditation does suggest that undertaking the processes necessary to attain Magnet status probably impacts retention as well. Unfortunately, the literature we reviewed does not reveal how these mechanisms impact the measured outcomes.

Quality improvement initiatives also tended to improve staff outcomes in the literature reviewed, although there were often some issues related to increased workload and apprehension about the implementation process. Interestingly, the only study to find strongly negative attitudes about quality improvement [50] examined dentists in the UK. Given that training seemed to increase acceptance of quality initiatives in several of the other studies and that many of the dentists felt they were lacking guidance, it is plausible that these dentists might have benefited from additional education on the process and benefits of evidence-based practice.

Making changes to how healthcare delivery is organized had mixed results; moves to team-based care sometimes resulted in increased stress or issues with role clarity [71], but a move to physician co-operative structures improved quality of life and stress levels among most respondents [73]. In two studies examining the effect of organization type (profit vs. non-profit and chain member vs. independent nursing homes), results were inconsistent. However, the Donoghue and Castle [76] study was based on a much larger sample than was Castle and Engberg's [77], which may account for the discrepant results.

Overall, the evidence is mixed with regard to how well the various governance mechanisms we reviewed work to create workforce change. More research is needed on each of these topics before we can draw strong conclusions about their effectiveness.

\section{What elements of governance mechanisms are critical to workforce outcomes?}

Each of the governance mechanisms we examined did have some effect on the workforce, and there are some critical elements common to all governance mechanisms that should be considered by health systems planning new initiatives. In sum, the elements that seem most important for successful change are: clear strategy and good leadership that focuses on communication and building trust; engagement of stakeholders from early development through implementation and into ongoing monitoring and refinement of new systems; organizational culture that supports the change and allocates resources to facilitate the process (e.g., funding for training); a reasonable pace for change; and flexibility to take account of local context.

Physician leadership and engagement are also important parts of any healthcare initiative. The value of getting and keeping physicians and other staff members involved in any governance mechanism should not be underestimated. Organizations wishing to begin any project should ensure that all relevant stakeholders are involved in planning and implementation, and should consider what they each value when designing the project.

A key topic that was touched on in many articles was the importance of clear, open communication during all stages of change. Communication from upper management about the organization's mission and values, along with a clear and reasoned explanation of the need for change were identified as crucial aspects of any kind of governance transformation.

There were a few critical elements unique to certain forms of governance. For shared governance, an important aspect to consider is whether shared governance is implemented in name only or whether providers truly feel in control of their practice. Two articles noted that shared governance might not develop as quickly or as fully as originally intended, and this should be taken into account when examining outcomes.

For quality-focused initiatives, staff seemed to be particularly accepting of and more consistent in implementing this kind of initiative when they received training on how to follow evidence-based guidelines and how to find and interpret research evidence. Training did tend to increase workload, stress, and time pressure, however, so management should consider ways to balance training with usual work requirements.

The literature included under Reorganization of Healthcare Delivery covered a wide range of topics, and thus we cannot conclude with certainty that various changes or types of organization are universally positive or negative. However, instilling trust in the workforce was an important factor in these changes. Organizations should also make sure to understand issues facing the workforce and take these into account when designing new care structures.

It is important to note that although many of the studies alluded to the importance of the above elements, we found no evidence that this had been empirically examined and thus cannot draw firm conclusions about whether they will be useful. That said, in general, elements such as stakeholder engagement, appropriate allocation of resources, strong leadership, clear communication, and training for providers should all be given consideration during the planning, implementation, and evaluation phases of any governance mechanism.

\section{How do health system governance mechanisms facilitate} workforce changes and contribute to health system change? There were some clear findings in the literature but, overall, the evidence on the impact of governance mechanisms on outcomes in the health workforce is patchy. This is partly explained by methodological weaknesses in the research we reviewed, much of which fails to 
account for the wide range of factors that may affect intervention implementation and outcomes in health systems. It is also a reflection of a lack of connection to theoretical models for change in the existing research e.g., $[89,90]$. There is also a case for more in-depth exploration of the contextual influences on transformational change in complex organizations. Richer, theoretically strong research is important for building the evidence base.

\section{Strengths and limitations of the review}

This systematic literature review had a number of strengths. It had a thorough methodological approach. Each abstract was screened according to preset criteria by four researchers and each full text article was rated and screened for relevancy and quality by at least two readers. Extractions and summaries were written and validated by two separate researchers to ensure all relevant information was included.

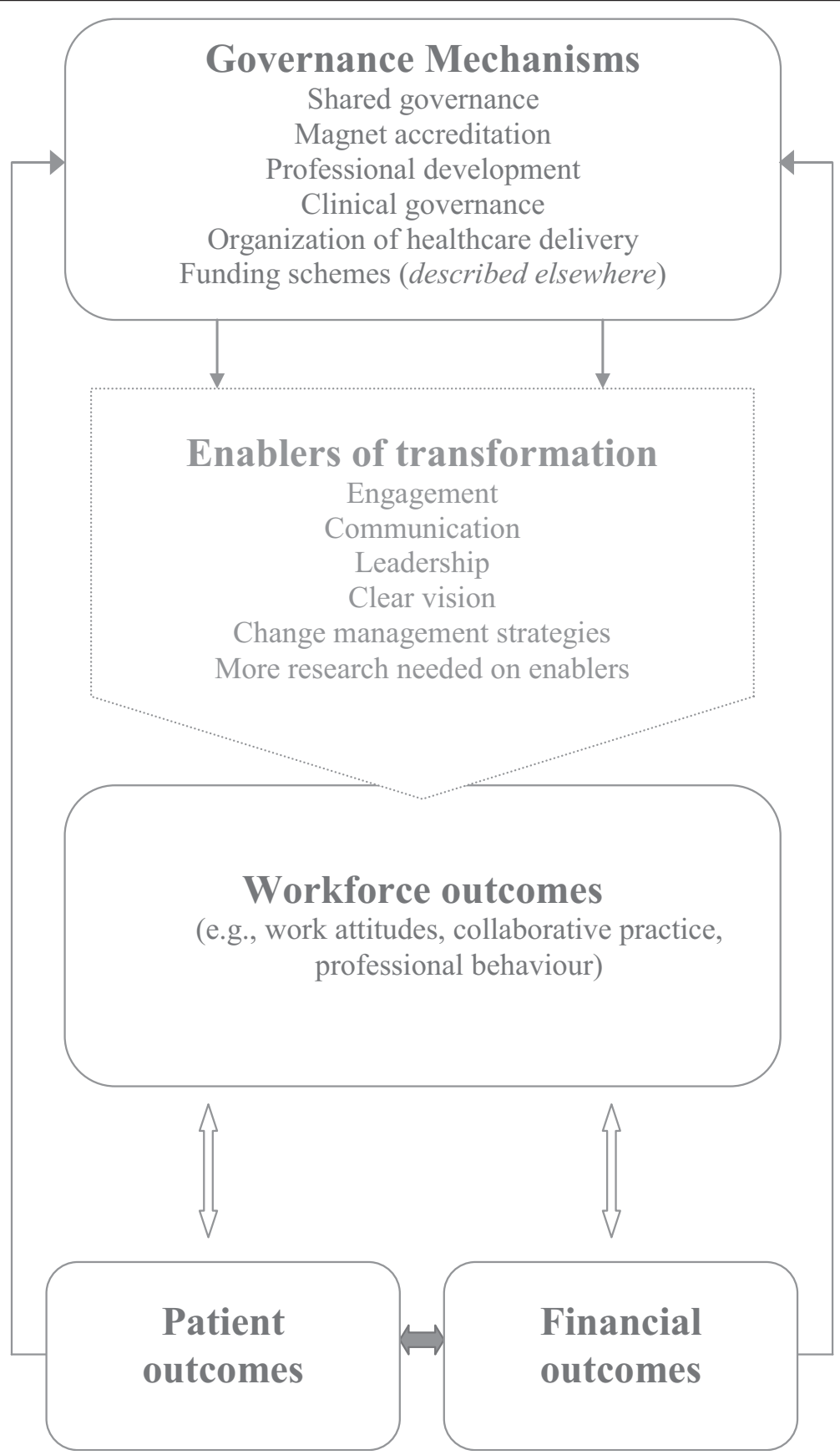

Figure 2 Workforce outcomes as mediator of relationship between governance mechanisms and patient or financial outcomes. 
Another strength of this review was the integration of guidance from knowledge users and health systems experts to ensure the relevancy and usability of the results. These experts were consulted regularly throughout the conception, search, synthesis, validation, and reporting phases of the project.

Finally, our literature search included not only published empirical literature, but also non-empirical articles and grey literature. This allowed us to examine government and health agency reports and consider expert advice on the topics under review.

The primary limitation of this review was the difficulty inherent in conducting a thorough literature search for governance mechanisms. Given the potential breadth of the topic, it is possible that some important topics or articles were missed despite the assistance provided by an experienced research librarian. We attempted to mitigate this limitation by asking our advisory committee to consider whether any other topics or key papers should be included. We also examined the bibliographies of included articles and retrieved empirical papers within our date range that seemed to address our research questions, and we searched for additional articles written by prominent authors.

The literature search was limited to articles from 2001 and newer, which may have excluded relevant literature. However, this ensured modern governance mechanisms were examined. We also limited our review to research from Canada, Sweden, the United Kingdom, the Netherlands, New Zealand, Australia, and the United States of America, which may also have excluded relevant literature.

Although the majority of literature did agree on key points the quality of papers we reviewed was not high enough to draw firm conclusions about many of the topics under consideration. There is a genuine need for high-quality research in most of the areas we covered. We were careful to eliminate papers with serious methodological flaws, but much of the remaining research did not include control groups, before-and-after designs, or other design elements that would allow us to infer causal linkages between governance, workforce outcomes, and health system change. There is a need for validated measurement tools, larger sample sizes, and the use of comparison groups. In-depth research on how local context impacts policy implementation processes would also help to develop the evidence base. Although research on change in health services organizations does exist $[88,90]$, the articles we reviewed did not incorporate this research to help explain the success or failure of their initiatives or to increase the odds of success in the planning stages. In addition, a segment of the research we reviewed was conducted by individuals working in the organization under study, which raises the question of conflict of interest. Unbiased, methodologically sound research underpinned by a strong theoretical base is sorely needed to allow users to draw strong conclusions about the effectiveness and suitability of any form of governance. Overall, the quality of evidence hampered our ability to draw strong inferences about the effectiveness of the governance structures and processes we reviewed.

\section{Conclusion}

The objective of this systematic review was to increase our understanding of the evidence relating health system governance to health workforce outcomes. The lack of high quality, empirical evidence making that link limits our ability to make firm recommendations but we suggest the following for consideration:

- Workforce should be considered as a mediating factor between governance mechanisms and health system outcomes. The literature we reviewed rarely considered both workforce and patient outcomes together. Governance mechanisms that are focused on patient, financial or other system outcomes should include explicit consideration, during the planning, implementation, and evaluation phases, of how the workforce will be affected in order to ensure that the workforce can and will carry out their work in the ways intended (see Figure 2 for an illustration).

- Decision-makers and researchers should work together to develop the evidence base to gain a more complete understanding of the consequences of various types of governance and the mechanisms through which they affect the workforce. Decisionmakers and researchers should both advocate for the collection of workforce-related outcomes of governance structures and processes to move research forward in this area.

\section{Additional files}

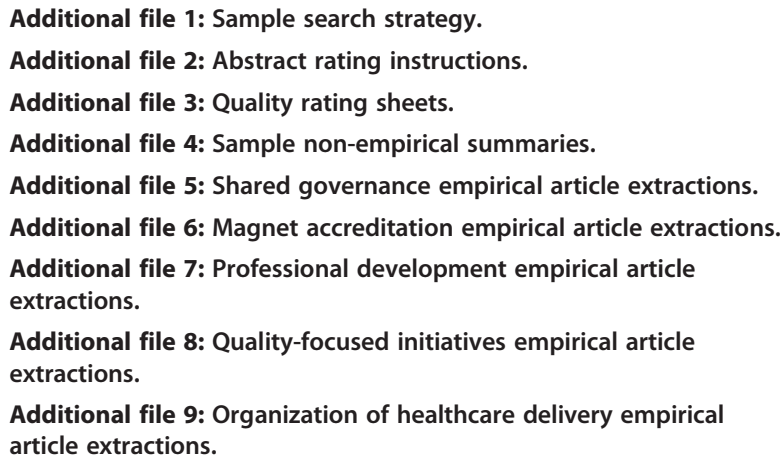

Additional file 9: Organization of healthcare delivery empirical article extractions.

\section{Competing interests}

The authors declare that they have no competing interests. 


\section{Authors' contributions}

ES and KJ designed the review. SH, GA, SM, ES, KJ screened abstracts. SH, GA, SM evaluated article inclusion and study quality. SH and GA wrote the first draft of the manuscript. All authors reviewed, revised, and approved the final manuscript.

\section{Acknowledgements}

The authors gratefully acknowledge the members of our advisory committee for their advice and constructive feedback throughout the project and Alberta Health Services for its in-kind contributions. This work was supported by Canadian Institutes of Health Research (CIHR) grant FRN: 119791.

Received: 8 August 2013 Accepted: 29 September 2014 Published: 4 October 2014

\section{References}

1. Health Council of Canada: An Environmental Scan of Current Views on Health Human Resources in Canada. Identified Problems, Proposed Solutions and gap Analysis. In Edited by El-Jardal F, Fooks C. http://www. healthcouncilcanada.ca/rpt_det.php?id = 132 .

2. Canadian Nurses Association: Tested solutions for eliminating Canada's registered nurse shortage. https://nursesunions.ca/sites/default/files/ rn shortage report_e.pdf.

3. World Health Organization: Increasing access to health workers in remote and rural areas through improved retention. In Edited by Dolea $C_{\text {, }}$ Stormont L, Zurn P, Shaw D, Braichet J-M. http://www.who.int/hrh/migration/ background_paper.pdf.

4. Levac D, Colquhoun H, O'Brien KK: Scoping studies; advancing the methodology. Implement Sci 2010, 5:69.

5. VanDeusen LC, Holmes S, Cohen A, Restuccia J, Cramer I, Shwartz M, Charns $\mathrm{M}$ : Transformational change in health care systems: an organizational model. Health Care Manage R 2007, 32:309-320.

6. Baranek P: A review of scopes of practice of health professions in Canada: a balancing Act. http://www.healthcouncilcanada.ca/rpt_det.php?id = 133 .

7. Health Professions Strategy and Practice: Clinical Workforce Strategic Plan 2011-2016. Edmonton (AB): Alberta Health Services; 2012

8. ACT Health: Workforce plan 2013-2018. http://www.health.act.gov.au/c/ health?a=sendfile\&ft=p\&fid=1907982279\&sid=

9. Health Workforce Australia: National health workforce innovation and reform strategic framework for action - background paper. http://www.hwa.gov. au/sites/uploads/wir-strategic-framework-background-paper-FinalFinal.pdf.

10. Ramanujam R, Rousseau DM: The challenges are organizational not just clinical. J Organ Behav 2006, 27:811-827.

11. Barbazza E, Tello JE: A review of health governance: definitions, dimensions and tools to govern. Health Policy 2014, 116:1-11.

12. de Savigny D, Adam T: Systems Thinking for Health Systems Strengthening: An Introduction. In Systems Thinking for Health Systems Strengthening. Edited by de Savigny D, Adam T. Geneva: World Health Organization; 2009.

13. Lewis M, Pettersson G: Governance in Health Care Delivery: Raising Performance. Washington (DC): The World Bank; 2009.

14. Brinkerhoff DW, Bossert TJ: Health governance: principal-agent linkages and health system strengthening. Health Policy Plan 2014, 29:685-693.

15. World Health Organization: Governance: http://www.who.int/healthsystems/ topics/stewardship/en/.

16. Hastings SE, Armitage GD, Mallinson S, Jackson K, Linder J, Misfeldt R, Suter E, Anderson B, Lewis S, Lorenzetti D, Mattern L, Moffatt M, Oelke N, Philippon D, Sproule J: Exploring the Relationship between Governance Models in Healthcare and Health Workforce Transformation: A Systematic Review. Calgary (AB): Alberta Health Services; 2013.

17. Popay J, Roberts H, Sowden A, Petticrew M, Britten N, Arai L, Roen K, Rodgers M: Developing guidance on the conduct of narrative synthesis in systematic reviews. J Epidemiol Comm Health 2005, 59(Suppl 1):A7.

18. Barden AM, Quinn Griffin MT, Donahue M, Fitzpatrick JJ: Shared governance and empowerment in registered nurses working in a hospital setting. Nurs Adm Q 2011, 35:212-218.

19. Erickson Jl, Hamilton GA, Jones DE, Ditomassi M: The value of collaborative governance/staff empowerment. J Nurs Adm 2003, 33:96-104.

20. Frith K, Montgomery M: Perceptions, knowledge, and commitment of clinical staff to shared governance. Nurs Adm Q 2006, 30:273-285.
21. Attree M: Nursing agency and governance: registered nurses' perceptions. J Nurs Manag 2005, 13:387-396.

22. Ellenbecker $\mathrm{CH}$, Samia L, Cushman MJ, Porell FW: Employer retention strategies and their effect on nurses' job satisfaction and intent to stay. Home Health Care Serv Q 2007, 26:43-58.

23. Latham $\mathrm{CL}$, Ringl K, Hogan M: Professionalization and retention outcomes of a university-service mentoring program partnership. J Prof Nurs 2011, 27:344-353.

24. Anderson EF: A case for measuring governance. Nurs Adm Q 2011, 35:197-203.

25. Batson $\mathrm{V}$ : Shared governance in an integrated health care network. $A O R N$ J 2004, 80:493-514.

26. Scott $L$, Caress $A$ : Shared governance and shared leadership: meeting the challenges of implementation. J Nurs Manag 2005, 13:4-12.

27. Winslow SA, Fickley S, Knight D, Richards K, Rosson J, Rumbley N: Staff nurses revitalize a clinical ladder program through shared governance. J Nurses Staff Dev 2011, 27:13-17.

28. Weston MJ: Managing and facilitating innovation and nurse satisfaction. Nurs Adm Q 2009, 33:329-344.

29. Smith Randolph D: Predicting the effect of extrinsic and intrinsic job satisfaction factors on recruitment and retention of rehabilitation professionals. J Healthcare Manage 2005, 50:49-60.

30. Jayawardhana J, Welton JM, Lindrooth R: Adoption of national quality forum safe practices by magnet hospitals. J Nurs Adm 2011, 41:350-356.

31. Upenieks $W$ : The interrelationship of organizational characteristics of magnet hospitals, nursing leadership, and nursing job satisfaction. Health Care Manage 2003, 22:83-98.

32. Brady-Schwartz DC: Further evidence on the magnet recognition program: implications for nursing leaders. J Nurs Adm 2005, 35:397-403.

33. Hess R, DesRoches C, Donelan K, Norman L, Buerhaus PI: Perceptions of nurses in magnet hospitals, non-magnet hospitals, and hospitals pursuing magnet status. J Nurs Adm 2011, 41:315-323.

34. Balogh $\mathrm{R}$, Cook M: Achieving magnet accreditation in the UK: a case study at rochdale NHS trust. J Nurs Manag 2006, 14:366-376.

35. New Zealand Ministry of Health: Health workforce development: an overview. http://www.health.govt.nz/publication/health-workforce-development-overview.

36. MacDonald CJ, Stodel EJ, Chambers LW: An online interprofessional learning resource for physicians, pharmacists, nurse practitioners, and nurses in long-term care: benefits, barriers, and lessons learned. Inform Health Soc Care 2008, 33:21-38.

37. Garrard J, Choudary V, Groom H, Dieperink E, Willenbring ML, Durfee JM, Ho SB: Organizational change in management of hepatitis C: evaluation of a CME program. J Cont Educ Health Prof 2006, 26:145-160.

38. George V, Burke $\sqcup$, Rodgers B, Duthie N, Hoffman ML, Koceja V, Kramer A, Maro J, Minzlaff P, Pelczynski S, Schmidt M, Westen B, Zielke J, Brukwitzki G, Gehring LL: Developing staff nurse shared leadership behaviour in professional nursing practice. Nurs Adm Q 2002, 26:44-59.

39. Prater $L$, Neatherlin JS: Texas nurses respond to mandatory continuing education. J Cont Educ Nurs 2001, 32:126-132.

40. Smith JE: Exploring the efficacy of continuing education mandates. JONA's Healthc Law Ethics Regul 2004, 6:22-31.

41. McCabe TJ, Garavan TN: A study of the drivers of commitment among nurses: the salience of training, development and career issues. J Eur Ind Train 2008, 32:528-568.

42. Narayanasamy A, Narayanasamy M: Advancing staff development and progression in nursing. Br J Nurs 2007, 16:384-388.

43. Murray J, Fell-Rayner H, Fine H, Karia N, Sweetingham R: What do NHS staff think and know about clinical governance? Clin Govern Int J 2004, 9:172-180.

44. Sweeney $\mathrm{G}$, Ellis $A$ : Making an impact: exploring delegates' perceptions of the clinical governance development programme. Clin Govern Int J 2003, 8:262-270.

45. Dean P, Faroogi A, McKinley RK: Quality improvement in general practice: the perspective of the primary healthcare team. Qual Prim Care 2004, 12:201-207.

46. Melnyk BM, Fineout-Overholt E, Giggleman M, Cruz R: Correlates among cognitive beliefs, EBP implementation, organizational culture, cohesion and job satisfaction in evidence-based practice mentors from a community health system. Nurs Outlook 2010, 58:301-308.

47. Sheaff R, Sibbald B, Campbell S, Roland N, Marshall M, Pickard S, Gask L, Rogers A, Halliwell S: Soft governance and attitudes to clinical quality in English general practice. J Health Serv Res Policy 2004, 9:132-138. 
48. Rosengren K, Hoglund PJ, Hedberg B: Quality registry, a tool for patient advantages - from a preventive caring perspective. J Nurs Manag 2012, 20:196-205.

49. Gerrish K, Ashworth P, Lacey A, Bailey J: Developing evidence-based practice: experiences of senior and junior clinical nurses. J Adv Nurs 2008, 62:62-73.

50. McCormick RJ, Langford JW: Attitudes and opinions of NHS general dental practitioners towards clinical governance. Brit Dent J 2006, 200:214-217.

51. Levin RF, Fineout-Overholt E, Melnyk BM, Barnes M, Vetter MJ: Fostering evidence-based practice to improve nurse and cost outcomes in a community health setting: a pilot test of the advancing research and clinical practice through close collaboration model. Nurs Adm Q 2011, 35:21-33.

52. Wallen GR, Mitchell SA, Melnyk B, Fineout-Overholt E, Miller-Davis C, Yates J, Hastings C: Implementing evidence-based practice: effectiveness of a structured multifaceted mentorship programme. J Adv Nurs 2010, 66:2761-2771

53. Som CV: Exploring the human resource implications of clinical governance. Health Policy 2007, 80:281-296.

54. Fitzgerald L, Ferlie E, Hawkins C: Innovation in healthcare: how does credible evidence influence professionals? Health Soc Care Comm 2003, 11:219-228.

55. Luxford K, Safran DG, Delbanco T: Promoting patient-centred care: a qualitative study of facilitators and barriers in healthcare organizations with a reputation for improving the patient experience. Int I Qual Health Care 2011, 23:510-515.

56. Mohide EA, Coker E: Toward clinical scholarship: promoting evidencebased practice in the clinical setting. J Prof Nurs 2005, 21:372-379.

57. Newhouse RP: Creating infrastructure supportive of evidence-based nursing practice: leadership strategies. Worldviews Evid Based Nurs 2007, 4:21-29.

58. Regan P: Critical issues in practice development: localism and public health reforms. Community Pract 2011, 84:32-35.

59. Lugon M: Clinical governance - from rhetoric to reality? Curr Paediatr 2005, 15:460-465.

60. Reinertsen JL, Gosfield AG, Rupp W, Whittington JW: Engaging Physicians in a Shared Quality Agenda. IHI Innovation Series White Paper. Cambridge, Massachusetts: Institute for Healthcare Improvement; 2007.

61. Spark Jl, Rowe S: Clinical governance: its effect on surgery and the surgeon. ANZ J Surg 2004, 74:167-170.

62. Wood J: Clinical supervision. Br J Perioper Nurs 2004, 14:151-156.

63. Forster AJ, Turnbull J, McGuire S, Ho ML, Worthington JR: Improving patient safety and physician accountability using the hospital credentialing process. Open Med 2001, 5:79-86.

64. Shaw C: Managing the Performance of Health Professionals. In Human Resources for Health in Europe. Edited by Dubois C, McKee M, Nolte E. England: Open University Press; 2006:98-115

65. Shortt S, Corbett S, Green M: Patient Safety \& Health System Governance: A Review of Approaches to Governing the Physician Sector. Kingston, ON: Centre for Health Services and Policy Research, Queen's University; 2006.

66. Webb V, Stark M, Cutts A, Tait S, Randle J, Green G: One model of healthcare provision lessons learnt through clinical governance. J Forensic Leg Med 2010, 17:368-373.

67. Jorm C, Kam P: Does medical culture limit doctors' adoption of quality improvement? Lessons from Camelot. J Health Serv Res Policy 2004, 9:248-251.

68. Jewell T, Wilkinson J: Health and social care regulation in Wales: an integrated system of political, corporate, and professional governance for improving public health. J R Soc Promot Health 2008, 128:306-312.

69. Rondeau KV: The adoption of high involvement work practices in Canadian nursing homes. Leadersh Health Serv 2007, 20:16-26.

70. Belling R, Whittock M, McLaren S, Burns T, Catty J, Jones IR, Rose D, Wykes T: Achieving continuity of care: facilitators and barriers in community mental health teams. Implement Sci 2001, 6:1-7

71. Lavoie-Tremblay M, Bonin J, Lesage A, Farand L, Lavigne GL, Trudel J: Implementation of diagnosis-related mental health programs: impact on health care providers. Health Care Manage 2011, 30:4-14.

72. Sicotte C, D'Amour D, Moreault M: Interdisciplinary collaboration within Quebec community health care centres. Soc Sci Med 2002, 55:991-1003.
73. O'Dowd TC, McNamara K, Kelly A, O'Kelly F: Out-of-hours co-operatives: general practitioner satisfaction with governance and working arrangements. Eur J Gen Pract 2006, 12:15-18.

74. Silvestro R, Silvestro C: Towards a model of strategic roster planning and control: an empirical study of nurse rostering practices in the UK national health service. Health Serv Manage Res 2008, 21:93-105.

75. Braithwaite J, Westbrook MT: A survey of staff attitudes and comparative managerial and non-managerial views in a clinical directorate. Health Serv Manage Res 2004, 17:141-166.

76. Donoghue C, Castle NG: Leadership styles of nursing home administrators and their association with staff turnover. Gerontologist 2009, 49:166-174.

77. Castle NG, Engberg J: Organizational characteristics associated with staff turnover in nursing homes. Gerontologist 2006, 46:62-73.

78. Aarons GA, Sommerfeld DH, Walrath-Greene CM: Evidence-based practice implementation: the impact of public versus private sector organizational type on organizational support, provider attitudes, and adoption of evidence-based practice. Implement Sci 2009, 4:1-13.

79. Smith C: Between the Scylla and charybdis: physicians and the clash of liability standards and cost cutting goals within accountable care organizations. Ann Health Law 2011, 20:165-203.

80. Korda H, Eldridge GN: ACOs, PCMHs, and health care reform: nursing's next frontier? Pol Polit Nurs Pract 2011, 12:100-103.

81. Howard C: Restructuring hospital-physician relationships for future success. Front Health Serv Manage 2003, 20:23-54.

82. Holloway E, Kusy M: Systems Approach to Address Incivility and Disruptive Behaviours in Health Care Organizations. In Organization Development in Healthcare: Conversations on Research and Strategies (Advances in Health Care Management, Volume 10). Edited by Wolf JA, Hanson H, Moir MJ, Friedman L, Savage GT. Bingley: Emerald Group Publishing Limited; 2011:239-265.

83. Scott C, Lagendyk L: Contexts and models in primary healthcare and their impact on interprofessional relationships. http://www.chsrf.ca/Libraries/ Commissioned_Research_Reports/ScottLagendyk-April2012-E.sflb.ashx.

84. Hinings CR, Casebeer A, Reay T, Golden-Biddle K, Pablo A, Greenwood R: Regionalizing healthcare in Alberta: legislated change, uncertainty, and loose coupling. Brit J Manage 2003, 14:S15-S30.

85. Freeman T, Walshe K: Achieving progress through clinical governance? A national study of health care managers' perceptions in the NHS in England. Qual Safe Health Care 2004, 13:335-343.

86. Paxton ES, Hamilton BG, Boyd VR, Hall BL: Impact of isolated clinical performance feedback on clinical productivity of an American surgical facility. J Am Coll Surg 2006, 202:737-745.

87. McCloskey BA, Diers DK: Effects of New Zealand's health reengineering on nursing and patient outcomes. Med Care 2005, 43:1140-1146.

88. Kramer M, Schmalenberg C, Maguire P, Brewer B, Burke R, Chmielewski L, Cox K, Kishner J, Krugman M, Meeks-Sjostrom D, Waldo M: Structures and practices enabling staff nurses to control their practice. Western $J$ Nurs Res 2008, 30:539-559.

89. Damschroder LJ, Aron DC, Keith RE, Kirsh SR, Alexander JA, Lowery JC: Fostering implementation of health services research findings into practice: a consolidated framework for advancing implementation science. Implement Sci 2009, 4:50

90. Greenhalgh T, Robert G, Macfarlane F, Bate P, Kyriakidou O: Diffusion of innovations in service organizations: systematic review and recommendations. Milbank Q 2004, 82(4):581-629.

doi:10.1186/1472-6963-14-479

Cite this article as: Hastings et al: Exploring the relationship between governance mechanisms in healthcare and health workforce outcomes: a systematic review. BMC Health Services Research 2014 14:479. 